Journal of Advanced Research in Fluid Mechanics and Thermal Sciences

\title{
Prediction of Fluid Behavior Using Generalized Hydraulic Calculation Method in Hydraulic Fractures
}

\author{
Siddharth Parekh ${ }^{1,}{ }^{*}$, Ali Pilehvari ${ }^{1}$, Robert Serth $^{1}$ \\ 1 Department of Chemical and Natural Gas Engineering, Texas A\&M University, Kingsville, Texas 77843, United States
}

\section{ARTICLE INFO ABSTRACT}

\section{Article history:}

Received 25 November 2020

Received in revised form 11 February 2021

Accepted 11 February 2021

Available online 15 March 2021

\begin{abstract}
Hydraulic fracturing has been used as one of the stimulation techniques to economically increase oil and gas production by creating small cracks in subsurface geologic formations to allow oil or gas to move toward a producing well. Hydraulics plays a vital role in many oil field operations including drilling, completion, fracturing and production. In the case of fracturing, however, the role of hydraulics becomes important since optimized hydraulics can minimize the cost and conversely, any miscalculations may cause problems such as the fluid loss or may potentially even lead to loss of the well. The current methods of the hydraulic calculation for non-Newtonian fluids necessitate determination of the robust model. This paper presented a new method for calculating pressure losses in the hydraulic fractures. The objective of this study was to develop the generalized model for hydraulic calculation for nonNewtonian fluid and run the case studies for the model validation. In the present work, detailed algorithm for the hydraulic calculation has been developed and then programmed in $\mathrm{C}^{++}$. The only input to the program is the raw rheological data, shear stress versus shear rate and the geometrical characteristics of the slit. Model validation with the new method has established a very small percentage difference between the values predicted by the model and experimental data. The results demonstrate that the new method is accurately predicting the pressure drop in both laminar and turbulent flow regimes. It is shown that the fluid behavior is more accurately represented using the new method than that with the standard fluid models available in the petroleum industry. Further validation and development to be carried out using experimental data for variety of fluid types.
\end{abstract}

Non-Newtonian fluid; pressure drop; hydraulic fracturing

\section{Introduction}

In an oil or gas well, hydraulic fracturing is used to create small cracks in subsurface geologic formations to allow oil or gas to move toward a producing well. Fractures and fracture networks are key conduits for migration of hydrothermal fluids, water and contaminants in groundwater systems, and oil and gas in petroleum reservoirs [1]. Fractures are also the principal pathways, through otherwise impermeable or low permeability rocks, for radioactive and toxic industrial wastes which may escape from underground storage repositories.

\footnotetext{
* Corresponding author.

E-mail address: siddh.parekh@gmail.com
}

https://doi.org/10.37934/arfmts.81.1.120130 
If we idealize flow along a discontinuity as being flow between a couple of smooth parallel plates and we assume the flow to be laminar, we reach the so-called "cubic law" [11]. The simplest model of flow through a rock fracture is the parallel plate model. This is the only fracture model for which an exact calculation of the hydraulic conductivity is possible [13]. Many authors agree that there is no alternative to the cubic law that is generally accepted yet [1]. In the new method development, we assume that the flow in the fracture takes place essentially parallel to the fracture's axis, overlooking any variations, say, of pressure, velocity or fluid concentration across the aperture or fracture's width.

The unexpected production behavior of many fields arising from an insufficient consideration of fracture effects on the flow, emphasized the need for better characterizing the fractures at various scales and transferring the meaningful part of this information to field simulation models. Hence, hydraulic calculation of non-Newtonian fluids in parallel plate geometry is very important for the design and operation of hydraulic fracturing of oil and gas wells and the hydraulic calculation for this geometry is thus of considerable practical interest.

Hydraulic calculation using Power-law, Bingham Plastic or similar other models, provide the estimates for simple conventional fluids. However, it is necessary to develop more powerful rheological models and more rigorous calculation methods which can predict the behavior of wide variety of fluids accurately. Therefore, in recent years, efforts have been made in providing more accurate hydraulics design tools for the fluids with unusual behavior. Since the computers are available on the rig sites, complexity of calculations is no longer a drawback. In this paper, a new and powerful rheological model, called Rational Polynomial (RP) model is introduced. Using the data from the literature, it is shown that the Rational-Polynomial model is capable of accurately representing the rheogram of virtually any time-independent fluid [14].

In conjunction with the RP model, a generalized hydraulic calculation method has also been developed using equivalent aperture. Rabinowitsch-Mooney equation is the basis for the hydraulic calculation that is independent of constitutive equation and fluid model. The prediction of the fluid behavior, derived using this novel method, was compared using several sets of experimental data. Based on the comparative analysis, the hydraulic calculation method adopted in this study, is shown to be very accurate as compared to the currently available standard techniques. The new method further to be developed based on the model validation results for the fluids with different nonNewtonian behavior.

\section{Rational Polynomial Modelling}

The rational polynomial function is suggested as a general correlating equation for rheometric data. The utility of the rational-polynomial model in hydraulic calculations is demonstrated using data for a variety of drilling fluids.

\subsection{Definition (Formula)}

A rational polynomial function, $\mathrm{RP}(\mathrm{x})$, can be written as [5]

$$
\mathrm{RP}(\mathrm{X})=\frac{\mathrm{P}_{0}+\mathrm{P}_{1} X+\mathrm{P}_{2} X^{2}+\mathrm{P}_{3} X^{3}+\ldots \ldots \ldots \ldots+\mathrm{P}_{\mathrm{k}} X^{k}}{1+\mathrm{Q}_{1} X+\mathrm{Q}_{2} X^{2}+\mathrm{Q}_{3} X^{3}+\ldots \ldots \ldots \ldots+\mathrm{Q}_{\mathrm{m}} X^{m}}
$$

where $\mathrm{k}$ and $\mathrm{m}$ are the degrees of the polynomials in the numerator and denominator, respectively. 
This equation can be applied to rheological model by equating $x$ with the shear rate, $\gamma$, and $\operatorname{RP}(x)$ with the shear stress, $\tau$.

\subsection{Application of RP Formula for Rheological Expression}

$$
\tau(\gamma)=\frac{\mathrm{P}_{0}+\mathrm{P}_{1} \gamma+\mathrm{P}_{2} \gamma^{2}+\mathrm{P}_{3} \gamma^{3}+\ldots \ldots \ldots \ldots+\mathrm{P}_{\mathrm{k}} \gamma^{k}}{1+\mathrm{Q}_{1} \gamma+\mathrm{Q}_{2} \gamma^{2}+\mathrm{Q}_{3} \gamma^{3}+\ldots \ldots \ldots \ldots+\mathrm{Q}_{\mathrm{m}} \gamma^{m}}
$$

where,

$P_{\mathrm{j}}$ : Ibf $\sec ^{\mathrm{j}} / \mathrm{ft}^{2}(\mathrm{j}=0,1,2 \ldots . \mathrm{k})$ and

$Q_{j:} \sec ^{i}(i=1,2 \ldots . m)$

\subsection{Physical Interpretation of the RP Model}

- Po represents the yield stress of the fluid.

- $P_{1}$ represents the zero-shear viscosity for fluid without a yield stress.

- $P_{k} / Q_{m}$ represents the infinite shear viscosity for $k=m+1$

For a realistic infinite shear behaviour, it is important to select a rational polynomial with the degree of numerator ( $k$ ) such that $k$ is greater by one than the degree of denominator $(m)$, i.e., $k=m+1$. Higher the value of $k$, better is the rational polynomial fit to the viscometric data. Dividing both sides of this equation by $\gamma$ gives

$$
\mu(\gamma)=\frac{\tau(\gamma)}{\gamma}=\frac{\frac{P_{0}}{\gamma}+P_{1}+P_{2} \gamma+P_{3} \gamma^{2}+\ldots \ldots \ldots \ldots+P_{k} \gamma^{k-1}}{1+Q_{1} \gamma+Q_{2} \gamma^{2}+Q_{3} \gamma^{3}+\ldots \ldots \ldots \ldots+Q_{m} \gamma^{m}}
$$

where, $\mu(\gamma)$ is the apparent viscosity at shear rate $\gamma$.

Consider now the limiting case of zero shear rate. From Eq. (2), if $P_{0} \neq 0$, then $\tau \rightarrow P_{0}$ as $\gamma \rightarrow 0$. Thus, $\mathrm{P}_{0}$ represents the yield stress of the fluid. Conversely, if $\mathrm{P}_{0}=0$, then from Eq. (3) it follows that $\mu \rightarrow P_{1}$ as $\gamma \rightarrow 0$. Hence, $P_{1}$ represents the limiting zero shear viscosity $\left(\mu=P_{1}\right)$ for a fluid without a yield stress.

In order to investigate the limiting case of infinite shear rate, it is convenient to divide both numerator and denominator in Eq. (2) by $\gamma^{k}$ to obtain

$$
\tau(\gamma)=\frac{P_{0} / \gamma^{k}+P_{1} / \gamma^{k-1}+P_{2} / \gamma^{k-2}+\cdots .+P_{k}}{1 / \gamma^{k}+Q_{1} / \gamma^{k-1}+Q_{2} / \gamma^{k-2}+\cdots . .+Q_{m} / \gamma^{k-m}}
$$

Dividing both sides of this equation by $\gamma^{n}$ gives

$$
\frac{\tau(\gamma)}{\gamma^{n}}=\frac{P_{0} / \gamma^{k}+P_{1} / \gamma^{k-1}+P_{2} / \gamma^{k-2}+\cdots . .+P_{k}}{1 / \gamma^{k-n}+Q_{1} / \gamma^{k-n-1}+Q_{2} / \gamma^{k-n-2}+\cdots . .+Q_{m} / \gamma^{k-n-m}}
$$

Now if $k=m$, it follows from Eq. (4) that $\tau \rightarrow P_{k} / Q_{m}$ as $\gamma \rightarrow \infty$. Likewise, if $k<m$, it follows that $\tau$ $\rightarrow 0$ as $\gamma \rightarrow \infty$. If $k=m+n$ where $n$ is a positive integer, it can be seen from Eq. (5) that $\tau \rightarrow\left(P_{k} / Q_{m}\right)$ $\gamma^{n}$ as $\gamma \rightarrow \infty$. Thus, for $n=1, \tau \rightarrow\left(P_{k} / Q_{m}\right) \gamma$ as $\gamma \rightarrow \infty$, and in this case $P_{k} / Q_{m}$ represents the limiting infinite shear viscosity $\left(\mu_{\infty}=P_{k} / Q_{m}\right)$ of the fluid. 
From the above discussion, it follows that the rational-polynomial model exhibits realistic infinite shear behaviour only for $k=m+1$. However, other combinations of $k$ and $m$ may provide perfectly adequate representations of fluid behaviour over practical ranges of shear rate. In general, the higher the value of $\mathrm{K}$, the better is the rational polynomial fit to the viscometric data.

Since the rational polynomial is a smooth function, it may be stated that depending on the relative values of the limiting shear viscosities, the model can represent shear thinning as well as shear thickening fluids.

A rational-polynomial model may exhibit other behaviour that is inconsistent with rheological data. If the polynomial in the denominator has a real root at any point, the model will predict an infinite shear stress at that point. Likewise, a rational polynomial may possess stationary points, i.e., points at which the derivative of $\tau$ with respect to $\gamma$ is zero.

Although some rheological models (e.g., the Reiner-Philippoff model) predict an inflection point, rheological data do not exhibit local maximum or minimum points [2]. The simple mathematical structure of the rational polynomials makes it easy to check for the occurrence of singularities and stationary points in the shear-rate range of interest. If such points are found, different values of $k$ and $\mathrm{m}$ can be used. In all cases studied to date, it has been possible to find values of $\mathrm{k}$ and $\mathrm{m}$ that give a good fit to the rheological data and for which no singularities or stationary points occur over a range of shear rates encompassing at least three times the range of the experimental data.

The number of parameters is small enough to be compatible with the size of most rheological data sets, although the rational polynomial model generally requires more parameters than the standard models to accurately represent the data.

\section{Hydraulic Calculation}

The calculations involve the determination of rational polynomial parameters and the prediction of pressure drop in the slit. Subramanian method has been used in the present work.

\section{- Step 1: Rational Polynomial Model Parameters}

After obtaining the rheometric data in the form of shear stress vs. shear rate, the first step is to determine the rational polynomial parameters that fit the data.

The program is developed to determine the model parameters. Since the hydraulic calculations may require extrapolation of the shear rate far beyond the range of the viscometric data, it is important to select a rational polynomial with proper infinite shear behaviour, i.e., with $\mathrm{k}=\mathrm{m}+1$ as noted previously. Choosing the degree of numerator as one greater than the degree of denominator generally gives a good fit to the viscometric data. After determining the rational polynomial model parameters, the pressure drop in the slit can be calculated as follows:

- Step 2: Integration of Rabinowitsch-Mooney equation

The Rabinowitsch-Mooney equation is integrated numerically to obtain a table of $\tau_{w}$ versus 6V/D. The following form of the equation is used:

$$
6 V / D=\left(3 / \tau_{w}{ }^{2}\right) \int_{0}^{\gamma_{W}} \tau \gamma(d \tau / d \gamma) d \gamma
$$


where, $\mathrm{D}=2 \mathrm{H}=$ Gap between the slit

$\mathrm{V}=$ average fluid velocity

$\tau=$ shear stress

$\tau_{\mathrm{w}}=$ shear stress at pipe wall

$\gamma=$ true shear rate

$\gamma_{w}=$ true shear rate at pipe wall

The RP model expression obtained in Step 1 is used to calculate $\tau$ and $d \tau / d \gamma$ in the integrand. The integration is carried out for values of $\gamma_{w}$ covering the range required for the hydraulic calculations. The integral is evaluated numerically using Romberg's algorithm for a range of $\gamma_{w}$ depending on the range of viscometric data. If the extrapolation beyond the range of the rheometric data (for turbulent flow calculations) is required, it is performed in this step.

- Step 3: Cubic spline fit

A cubic spline is fitted to $\ln \tau_{w}$ versus $\ln (6 V / D)$ data obtained from Step 2 . This spline is used in all subsequent calculations. Thus, the RP model is used only to perform the numerical integration in Step 2.

- Step 4: Calculation of $\tau_{\mathrm{w}}$ and $\mathrm{n}^{\prime}$

For a given flow rate, regardless of the flow regime, the value of $6 \mathrm{~V} / \mathrm{D}$ is first calculated. Using this value, the corresponding shear stress $\left(\tau_{w}\right)$ and the slope $\left(n^{\prime}\right)$ are obtained from the cubic spline. The relationship between $6 V / D, \tau_{w}$, and $n$ ' for the generalized power- law model is given by Metzner and Reed according to Eq. (7) and Eq. (8).

$$
\begin{aligned}
& \tau_{W}=K^{\prime}(6 V / D)^{n^{\prime}} \\
& n^{\prime}=\frac{d \ln \tau_{W}}{d \ln (6 V / D)}
\end{aligned}
$$

As Eq. (8) shows, $n^{\prime}$ is obtained by differentiating the cubic spline at the given value of 6V/D.

- Step 5: Generalized Reynolds number, $\mathrm{N}_{\mathrm{Re}, \mathrm{G}}$

The generalized Reynolds number is calculated from Eq. (11) according to Dodge and Metzner.

$$
N_{\mathrm{Re}, G}=\frac{\mathrm{D}(6 \mathrm{~V} / \mathrm{D}) \mathrm{V} \rho}{\tau_{w}}
$$

The volumetric flow rate of the fluid and the pipe dimensions are specified; hence the average velocity can be computed. The initial trial value for the wall shear stress, $\tau_{w}$ at the specified value of $6 \mathrm{~V} /$ Deq is obtained from the interpolating spline of $\tau_{\mathrm{w}} \mathrm{Vs} .6 \mathrm{~V} /$ Deq data. 
The values of $\mathrm{n}^{\prime}$ and $\mathrm{K}^{\prime}$ at this $\tau_{\mathrm{w}}$ can be computed from the relations

$$
\begin{aligned}
& n^{\prime}=\frac{d\left[\ln \tau_{W}\right]}{d[\ln (6 V / D)]} \\
& \mathrm{K}^{\prime}=\frac{\tau_{w}}{(6 V / D)^{\mathrm{n}^{\prime}}}
\end{aligned}
$$

Note that $\mathrm{n}^{\prime}$ and $\mathrm{K}^{\prime}$ are the slope and intercept, respectively, of a log-log plot of $\tau_{\mathrm{w}} \mathrm{vs}$. $(6 \mathrm{~V} / \mathrm{D})$ called the flow curve. The value of $n^{\prime}$ is obtained by numerically differentiating the spline function of $\tau_{\mathrm{w}}$.

In the present work Reynolds number used is modified Metzner-Reed Reynolds number and is defined as [5]

$$
N_{\mathrm{Re}, G}=\frac{8 D^{\mathrm{n}^{\prime}} V^{2-\mathrm{n}^{\prime}} \rho}{\mathrm{K}^{\prime} g_{c} 6^{\mathrm{n}^{\prime}}}
$$

The above Reynolds number is used in the pressure drop calculations and the flow regime is determined using this Reynolds number and the friction factor.

- Step 6: Friction factor calculation

Based on the values of $\mathrm{N}_{\mathrm{Re}, \mathrm{G}}$ and $\mathrm{n}^{\prime}$, two friction factors are calculated, one for laminar flow $\left(\mathrm{f}_{\mathrm{L}}\right)$, Eq. (13), and one for turbulent flow $\left(f_{T}\right)$, Eq. (14).

$$
f_{L}=\frac{16}{N_{\mathrm{Re}, G}}
$$

The turbulent friction factor is calculated using the friction factor correlation given by Dodge and Metzner as

$$
\sqrt{\frac{1}{f_{t}}}=\frac{4.0}{\left(\mathrm{n}^{\prime}\right)^{0.75}} \log \left\lfloor N_{\mathrm{Re}, G}\left(f_{t}\right)^{1-\frac{\mathrm{n}^{\prime}}{2}}\right\rfloor-\frac{0.40}{\left(\mathrm{n}^{\prime}\right)^{1.2}}
$$

The turbulent flow friction factor formula used is a combination of the Dodge-Metzner equation, and Colebrook's equation, which includes the roughness effect. Derivation and details of the formula were given by Reed and Pilehvari [3,4,9].

\section{- Step 7: Determination of Flow Regime}

The two friction factors calculated in the previous step for laminar and turbulent flow regimes are compared, and whichever is larger is assumed to be the correct value and determines the flow regime, laminar or turbulent. 
- Step 8: Calculation of New estimated Value of $\tau_{\mathrm{w}}$

The just selected friction factor, $f$, is used to obtain a new estimate of $\tau_{w}$, the shear stress at the pipe wall, from the defining equation for friction factor

$\tau_{w}=\frac{f \rho V^{2}}{2 g_{c}}$

- Step 9: Calculation of Pressure drop

The pressure drop is calculated from the shear stress at the slit wall

$\Delta P=\frac{2 \tau_{w} L}{D}$

where, $\mathrm{D}=2 \mathrm{H}=$ Gap between parallel plates.

\section{Results and Discussions}

In the present work, $\mathrm{C}^{++}$program has been modified and deveploped for the slit flow [6]. The pressure drop predictions (Figure 1 to Figure 5) for slit flow are compared with that of concentric annulus flow by adjusting the dimensions equivalent to each other. The relation between dimensions can be expressed as follows

$2 H=\frac{\left(D_{2}-D_{1}\right)}{2}$

$W=\Pi \frac{\left(D_{1}+D_{2}\right)}{2}$

where,

$D_{1}=$ Inside Diameter

$D_{2}=$ Outside Diameter

The average percentage difference in pressure drop predictions (Figure 1 to Figure 5) for Water/Gel with five different dimensions of annulus are analysed [12].

- Based on the results tabulated in Table 1, there are not much difference in pressure drop prediction range from $0.14 \%$ to $1.41 \%$ for different geometries. Here, the $D_{1} / D_{2}$ ratio ranges from 0.88 to 0.62 and $\mathrm{W} / 2 \mathrm{H}$ ratio ranges from 14 to 48 .

- As we increase the gap between two plates or increase the distance between two concetric cylinder, \% pressure drop difference increases.

- Geometry of $6.407 "-4.0^{\prime \prime}$ gives highest difference in pressure drop between two flows. The large difference in pressure drop exists at the initial value of flow rate when the pressure drop is very low. 


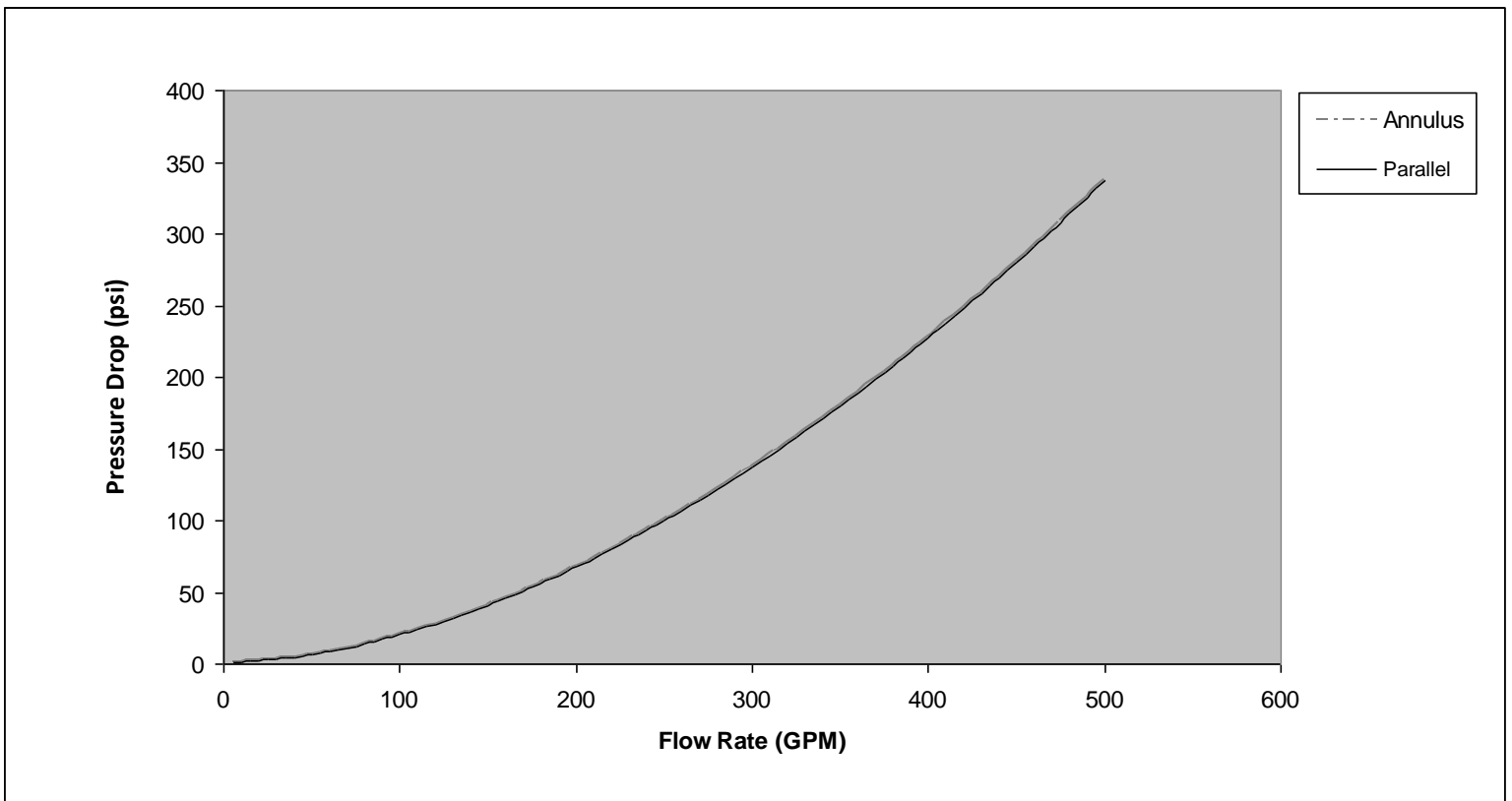

Fig. 1. Pressure drop prediction for 4.563" - 4.0" Concentric Annulus and equivalent Parallel Plate (Water/Gel)

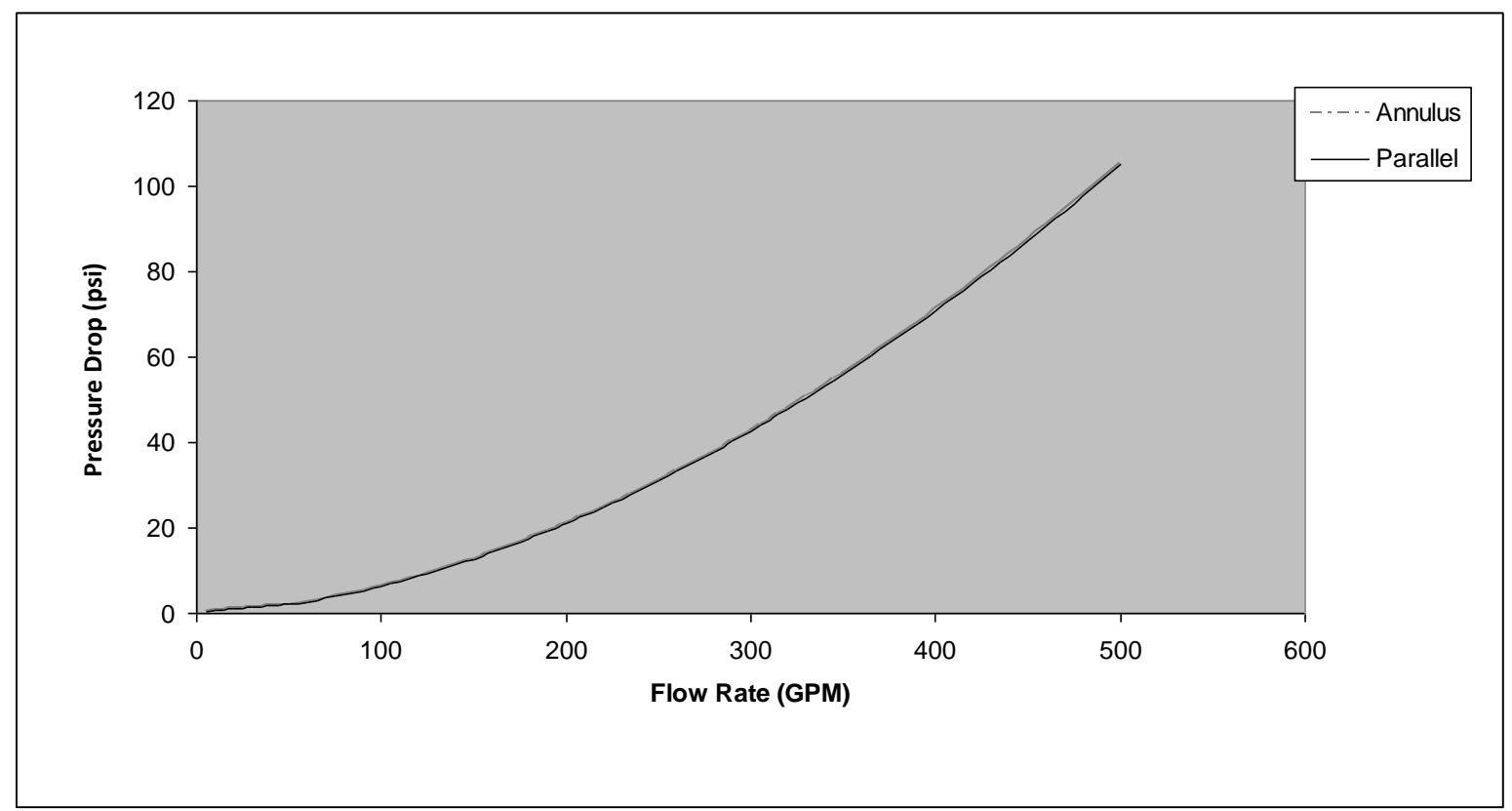

Fig. 2. Pressure drop prediction for 4.813" - 4.0" Concentric Annulus and equivalent Parallel Plate (Water/Gel) 


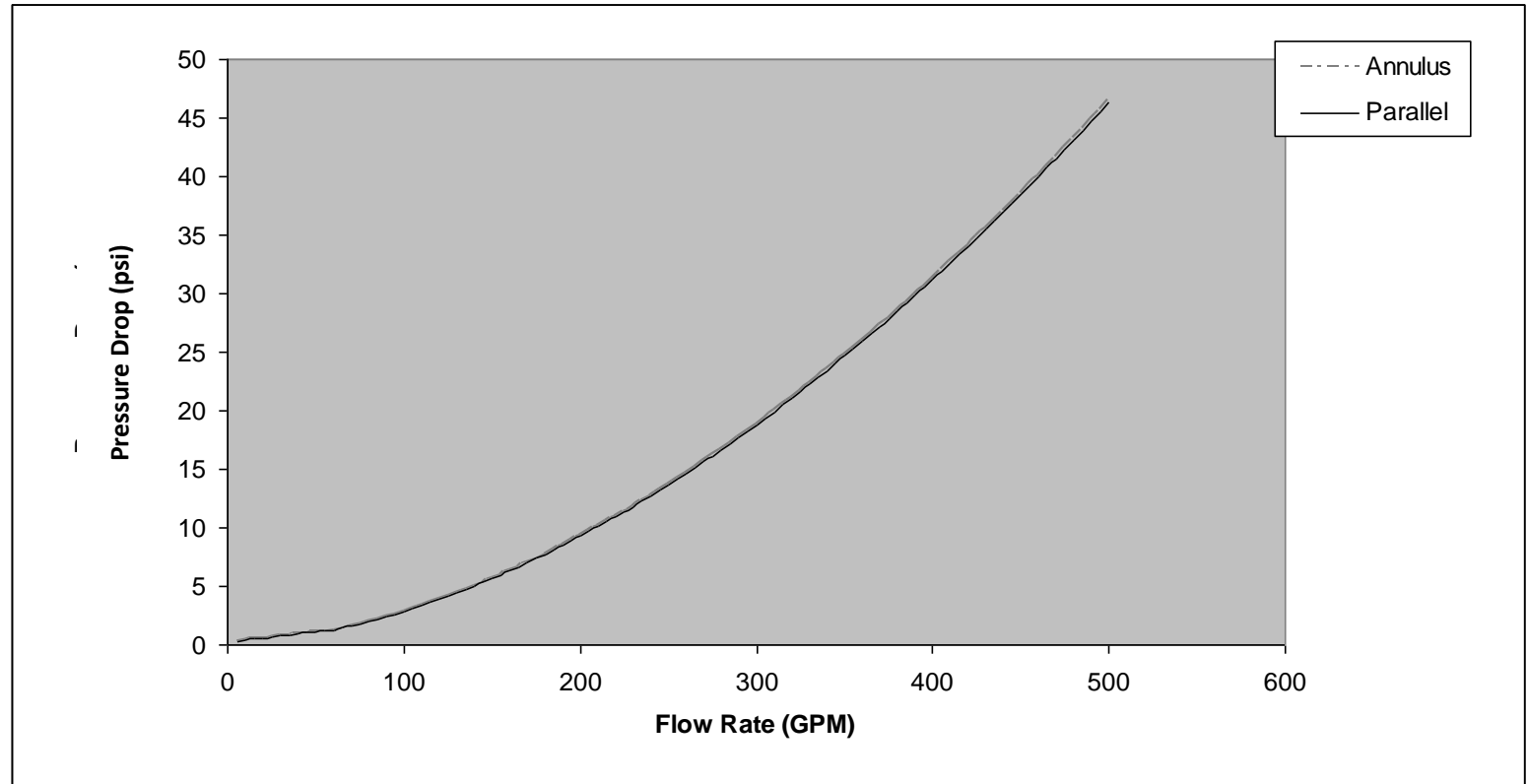

Fig. 3. Pressure drop prediction for 5.047" - 4.0" Concentric Annulus and equivalent Parallel Plate (Water/Gel)

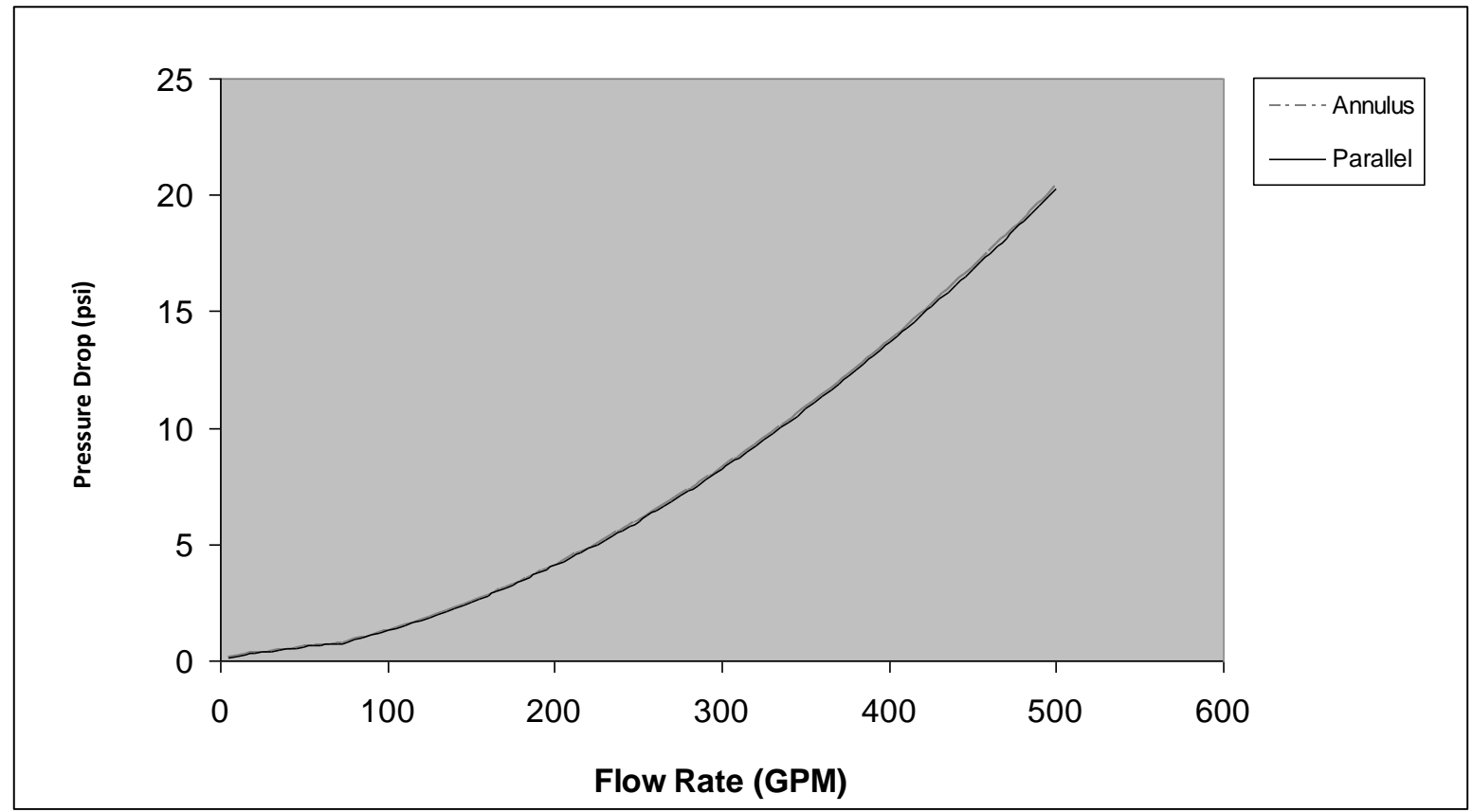

Fig. 4. Pressure drop prediction for 5.345" - 4.0" Concentric Annulus and equivalent Parallel Plate Water/Gel) 


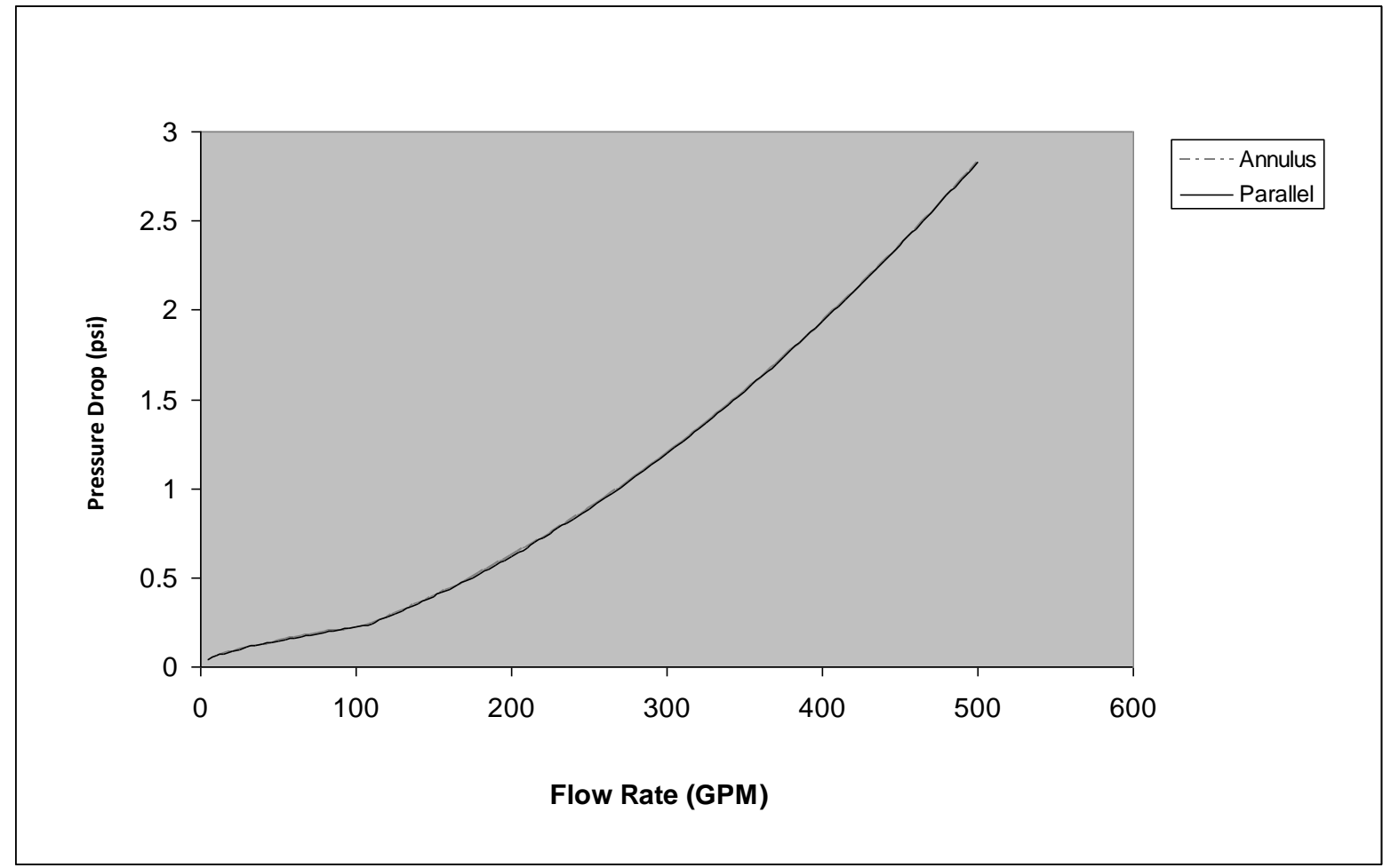

Fig. 5. Pressure drop prediction for 6.407" - 4.0" Concentric Annulus and equivalent Parallel Plate (Water/Gel)

\section{Table 1}

The average percentage difference in pressure drop predictions for Water/Gel with five different dimensions of annulus [12]

\begin{tabular}{|c|c|c|c|c|c|c|}
\hline \multirow[t]{3}{*}{ No. } & \multicolumn{4}{|c|}{ Dimension (inch) } & \multirow{2}{*}{\multicolumn{2}{|c|}{$\begin{array}{l}\text { \% Pressure Drop } \\
\text { Difference } \\
\text { Water/Gel }\end{array}$}} \\
\hline & \multicolumn{2}{|c|}{ Annulus } & \multicolumn{2}{|l|}{ Parallel } & & \\
\hline & D2 & D1 & $2 \mathrm{H}$ & W & Average & Maximum \\
\hline 1. & 4.563 & 4.0 & 0.2815 & 13.44391 & $0.14 \%$ & $2.82 \%$ \\
\hline 2. & 4.813 & 4.0 & 0.4065 & 13.83641 & $0.21 \%$ & $3.43 \%$ \\
\hline 3. & 5.047 & 4.0 & 0.5235 & 14.20379 & $0.34 \%$ & $3.99 \%$ \\
\hline 4. & 5.345 & 4.0 & 0.6725 & 14.67165 & $0.56 \%$ & $5.08 \%$ \\
\hline 5. & 6.407 & 4.0 & 1.2035 & 16.33899 & $1.41 \%$ & $11.40 \%$ \\
\hline
\end{tabular}

\section{Conclusion}

Hydraulic calculation of non-Newtonian fluids in parallel plate geometry is very important for the design and operation of hydraulic fracturing of oil and gas wells and the hydraulic calculation for this geometry is thus of considerable practical interest.

- These effective results show that the program delivers the accurate results. It means that RP model can represent the performance of the slit flow effectively and correctly.

- Model has been checked for feasibility of pressure drop predictions for slit flow by comparing pressure drop predictions for annuli and slit. The results show that slit flow predictions are fairly similar to those of annuli.

Based on the comparative analysis, the new hydraulic calculation method adopted in this study, is shown to be very accurate. As future development, this novel method will be validated further using the experimental data for the fluids with different properties. 


\section{Acknowledgement}

I would like to acknowledge with sincere gratitude to Dr. Ali Pilehvari and Dr. Robert Serth for their support and guidance throughout the project.

\section{References}

[1] Berkowitz, Brian. "Characterizing flow and transport in fractured geological media: A review." Advances in Water Resources 25, no. 8-12 (2002): 861-884. https://doi.org/10.1016/S0309-1708(02)00042-8

[2] Bird, R. B., W. E. Stewart, and E. N. Lightfood. Transport Phenomena-Revision 2nd. John Wiley \& Sons, New York (1960).

[3] Dodge, D. W., and A. B. Metzner. "Turbulent flow of non-Newtonian systems." AlChE Journal 5, no. 2 (1959): 189204. https://doi.org/10.1002/aic.690050214

[4] Govier, George Wheeler, and Khalid Aziz. "The flow of complex mixtures in pipes." Van Nostrand-Reinhold, New York (1972): 98.

[5] Hanna, Owen T., and Orville C. Sandall. Computational methods in chemical engineering. Prentice Hall, 1995.

[6] Kumar, A. "Rational Polynomial Model for non-Newtonian Fluids", Master Thesis diss., Texas A\&M UniversityKingsville (1997).

[7] Metzner, A. B., and J. C. Reed. "Flow of non-newtonian fluids-correlation of the laminar, transition, and turbulentflow regions." AIChE Journal 1, no. 4 (1955): 434-440. https://doi.org/10.1002/aic.690010409

[8] Kumar, A., S. Saboo, S. Sheth, A. Pilehvari, and R. Serth. "Correlation of rheometric data and hydraulic calculations using rational polynomials." Chemical Engineering Communications 183, no. 1 (2000): 99-117. https://doi.org/10.1080/00986440008960504

[9] Reed, T. D., and A. A. Pilehvari. "A new model for laminar, transitional, and turbulent flow of drilling muds." In SPE Production Operations Symposium. Society of Petroleum Engineers, 1993. https://doi.org/10.2118/25456-MS

[10] National Research Council. "Committee on fracture characterization and fluid flow." Rock Fractures and Fluid Flow: Contemporary Understanding and Applications 316 (1996).

[11] Snow, David Tunison. "A parallel plate model of fractured permeable media." PhD. Thesis, University of California (1965).

[12] Subramanian, R. "A study of pressure loss correlations of drilling fluids in pipes and annuli." PhD diss., University of Tulsa, 1995.

[13] Sarkar, Sudipta, M. Nafi Toksoz, and Daniel R. Burns. Fluid flow modeling in fractures. Massachusetts Institute of Technology. Earth Resources Laboratory, 2004.

[14] Pilehvari, Ali, Robert Serth, and Vishal Lagad. "Generalized hydraulic calculation method using rational polynomial model." In SPE Annual Technical Conference and Exhibition. Society of Petroleum Engineers, 2001. https://doi.org/10.2118/71403-MS 